\title{
The timescale and extent of thermal expansion of the global ocean due to climate change
}

\author{
S. Marčelja \\ Department of Applied Mathematics, Research School of Physics and Engineering, The Australian National University, \\ Canberra, Australia
}

Faculty of Science, University of Split, Split, Croatia

Received: 10 November 2009 - Published in Ocean Sci. Discuss.: 3 December 2009

Revised: 2 February 2010 - Accepted: 3 February 2010 - Published: 5 February 2010

\begin{abstract}
With recently improved instrumental accuracy, the change in the heat content of the oceans and the corresponding contribution to the change of the sea level can be determined from in situ measurements of temperature variation with depth. Nevertheless, it would be favourable if the same changes could be evaluated from just the sea surface temperatures because the past record could then be reconstructed and future scenarios explored. Using a single column model we show that the average change in the heat content of the oceans and the corresponding contribution to a global change in the sea level can be evaluated from the past sea surface temperatures. The calculation is based on the time-dependent diffusion equation with the known fixed average upwelling velocity and eddy diffusivity, as determined from the steadystate limit. In this limit, the model reduces to the 1966 Munk profile of the potential temperature variation as a function of depth.

There are no adjustable parameters in the calculation and the results are in good agreement with the estimates obtained from the in situ data. The method allows us to obtain relevant timescales and average temperature profiles. The evaluation of the thermosteric sea level change is extended back to the beginning of accurate sea surface temperature records. The changes in sea surface temperature from 1880 until the present time are estimated to have produced a thermosteric sea level rise of $35 \mathrm{~mm}$. Application to future IPCC scenarios gives results similar to the average prediction of more complex climate models.
\end{abstract}

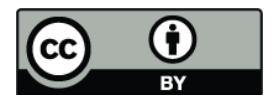

Correspondence to: S. Marčelja (stjepan.marcelja@anu.edu.au)

\section{Introduction}

Increasingly accurate estimates of the change in ocean heat content have become available in the past two years (Domingues et al., 2008; Levitus et al., 2009) thus providing information on the major part of the global heat accumulation. The temperatures of the ocean interior reflect the variations of the past sea surface temperatures up to the present time. Improving the understanding of this unknown functional relationship should provide insight into the mechanism of global warming resulting from climate change. The resulting global thermosteric sea level increase is an important component in the predictions of future climate change scenarios.

Simple models that use spatial averaging over the whole or parts of the global ocean have in the past been successful in intuitive interpretation of the basic features of ocean profiles. More recently, attempts have been made to apply simple global average models to sea level modeling (Rahmstorf, 2007; Grinsted et al., 2009; Siddal et al., 2009). Modeling just the global ocean heat content increase and the thermosteric part of the sea level rise is a simpler task, which can be put on a more quantitative basis and integrated with existing knowledge. We use here a time-dependent version of the Munk (1966) classic steady state analysis of potential temperature and salinity variation as a function of depth and show that it provides an accurate description of average global ocean heat content and thermosteric sea level change.

The calculation by Munk (1966) is based on the steady state balance between the turbulent diffusion and upwelling. We explore the time dependence of the average ocean warming using the mixing equation, which combines vertical diffusion and advection (see e.g. Stewart, 2009): 
$\frac{\partial \psi(z, t)}{\partial t}=A_{z} \frac{\partial^{2} \psi(z, t)}{\partial z^{2}}-W \frac{\partial \psi(z, t)}{\partial z}$,

where $\psi(z, t)$ is the potential temperature or tracer density, $A_{z}$ the vertical eddy diffusivity and $W$ the mean vertical velocity. Munk (1966) reviewed the data and determined the characteristic length $A_{z} / W$ from a fit to the thermocline in the Pacific between $1 \mathrm{~km}$ and $4 \mathrm{~km}$ depth and $A_{z} / W^{2}$ from the vertical distribution of ${ }^{14} \mathrm{C}$. The resulting values $A_{z} \approx 1.3 \times 10^{-4} \mathrm{~m}^{2} / \mathrm{s}$ and $W \approx 1.2 \mathrm{~cm} /$ day are used throughout this work.

Most of the changes in the ocean heat content resulting from climate change are presently occurring in the top $1000 \mathrm{~m}$. If the simple advection-diffusion model of Eq. (1) were valid in this region, changes due to the surface warming could be calculated independently of the established steadystate thermocline because Eq. (1) is linear and superposition holds. In the subsequent sections we show that using the advection-diffusion model with Munk's average values for $A_{z}$ and $W$ to calculate the average ocean heat content and sea level change produces accurate results. Apart from the arbitrary choice of the reference zero value for the change in heat content and sea level, we need no adjustable parameters. The applicability of the advection-diffusion model with Munk's values for $A_{z}$ and $W$ is thus extended to the calculation of average global temporal variations, including those occurring in the upper ocean.

In the next section we begin the analysis with a discussion of the response to a step change in surface temperature. The solution is required in the subsequent section, which uses sea surface temperatures as measured from 1880 to calculate the change in ocean heat content and thermosteric sea level. The approach is validated by the very good agreement with the findings based on in situ data. The report ends with a brief discussion, including the evaluation of the future steric sea level increase predicted from present-day temperatures and from standard IPCC scenarios.

\section{Response to a step change in sea surface temperature}

Properties of the solution for a step change in surface temperature are interesting because they indicate the time scales of the problem and the ultimate steric sea level change for each degree of surface warming. Let us assume the ocean is in the natural steady state temperature distribution and make a step change in surface temperature at time $t=0$, so that $\psi(z, t)=0$ for $t<0$ and $\psi(0, t)=1$ for $t>0$. The depth $z$ is measured downwards from the surface and the velocity $W$ is hence negative. The solution of Eq. (1) for this boundary condition is found using Laplace transforms (Ogata, 1970; Lee, 1999)

$\psi(z, t)$

$=\frac{1}{2} \exp \frac{W z}{2 A_{z}}\left(\exp \frac{-W z}{2 A_{z}} \operatorname{erfc} \frac{z-W t}{2 \sqrt{A_{z} t}}+\exp \frac{W z}{2 A_{z}} \operatorname{erfc} \frac{z+W t}{2 \sqrt{A_{z}}}\right)$, where erfc is the complementary error function. In the steady state limit $t \rightarrow \infty$, Eq. (1) reduces to the Munk profile $\psi(z, \infty)=\exp \left(-W z / A_{z}\right)$. The boundary condition at the bottom of the ocean is not included, as it has very little effect over a time scale of 1000 years or less. Working in the limit of infinite depth does not lead to an observable difference in the results.

Integrating $\psi(z, t)$ over the depth gives a characteristic length $\lambda(t)$, which can be interpreted as the extent of the penetration of the change from the surface

$$
\begin{aligned}
\lambda(t) & =\int_{0}^{\infty} \psi(z, t) d z \\
& =\sqrt{\frac{A_{z} t}{\pi}} \exp \frac{-W^{2} t}{4 A_{z}}+\frac{W t}{2}+\left(\frac{A_{z}}{W}+\frac{W t}{2}\right) \operatorname{erf} \frac{W t}{2 \sqrt{A_{z} t}} .
\end{aligned}
$$

The Munk limiting value is $\lambda(\infty)=-A_{z} / W \approx 0.9 \mathrm{~km}$.

In applications addressing the effects of temperature change at the ocean surface, the calculated average potential temperature change profile must be added to the already existing steady-state thermocline profile. The approach to the limit as a function of depth and time after a step change is shown in Fig. 1.

The approach towards the new steady state can be examined by calculating the total change in the heat content of the oceans as a function of time. We integrate the temperature change profile over all depths and use $4186 \mathrm{~J} /(\mathrm{kg} \mathrm{K})$ for the average specific heat and $3.61 \times 10^{8} \mathrm{~km}^{2}$ for the global ocean surface area. The result is shown in Fig. 2, and the steady state limit is $1.4 \times 10^{24} \mathrm{~J}$ of absorbed heat for a $1 \mathrm{~K}$ increase in the surface temperature. The slow approach to the limit is reminiscent of simple diffusion, where the diffusion length, defined as $L=\sqrt{4 D t}$, increases as the square root of time. We estimate the characteristic time as $\tau=\lambda^{2}(\infty) /\left(4 A_{z}\right)=$ $A_{z} /\left(4 W^{2}\right) \approx 50$ years. During that time nearly half of the heat transfer has been completed. The diffusion gradually becomes slower, and the change in the heat content beyond 200 years can be accurately fitted with an exponential function with the characteristic time of 280 years.

The corresponding thermosteric increase in the global sea level needs to take into account the variation of thermal expansion coefficient with temperature and salinity. We used the average global ocean profiles of temperature and salinity (Levitus, 1982) and an accurate 25-term approximation to the equation of state for seawater (McDougall et al., 2002) to evaluate the average thermal expansion coefficient as a function of depth. The values fall rapidly from $\alpha=2.4 \times 10^{-4} / \mathrm{K}$ at the surface to $\alpha=0.8 \times 10^{-4} / \mathrm{K}$ at $5000 \mathrm{~m}$ depth. The calculated change in the sea level as a function of time is also shown in Fig. 2. Compared to the variation in the heat content, the sea level increases more rapidly in the initial period after a temperature change when heat is absorbed closer to the surface, and more slowly after hundreds of years when the heat penetrates into deeper ocean where the coefficient of 


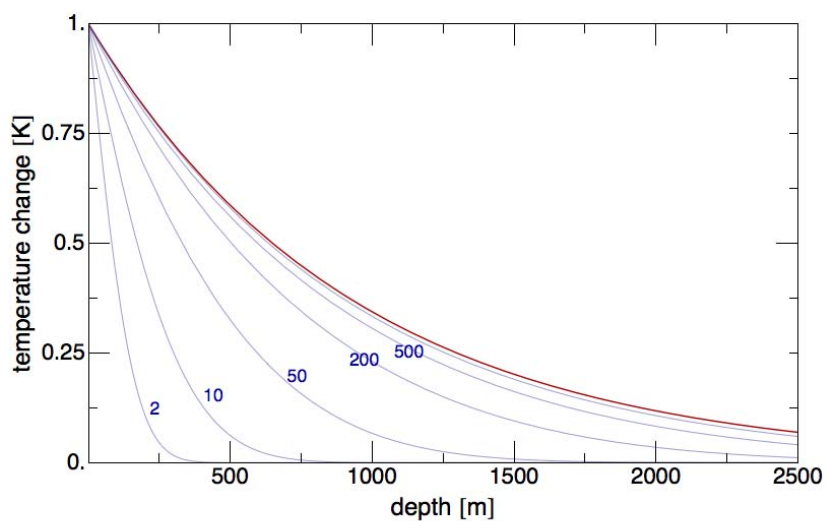

Fig. 1. Average potential temperature change profiles after a step change of temperature at the surface. The evaluation uses Munk's values for the vertical diffusivity and vertical drift velocity. The time after the change in years is shown in the figure for the first five curves. The top red curve is the steady-state limit and the one immediately below corresponds to 1000 years.

thermal expansion is small. The asymptotic value is $13 \mathrm{~cm}$ for each degree of surface warming.

\section{Evaluation of the heat content and sea level change from surface temperatures}

The measured variation in global sea surface temperatures may be considered as a sum of small step changes. Exploiting the superposition property of Eq. (1) we may evaluate a composite change by adding a number of solutions for a step change. If $\operatorname{SST}(t)$ is the externally given sea surface temperature, a series of small changes is multiplied by the step change solution Eq. (2) and integrated over time to obtain the change in temperature profile

$\vartheta(z, t)=\int_{0}^{t} \frac{d \operatorname{SST}\left(t^{\prime}\right)}{d t^{\prime}} \psi\left(z, t-t^{\prime}\right) d t^{\prime}$.

The temperature change profile Eq. (4) was evaluated in discrete time. We use the step of one year corresponding to annual values of mean sea surface temperatures at the centre of the time step. Denoting the average sea surface temperature for the year $i$ as $\operatorname{SST}(i)$ and using the solution for a step change, Eq. (2), the composite solution $\vartheta(z, n, k)$ describing the temperature change in year $n$ resulting from the sea surface temperature changes beginning in the year $k$ is evaluated as

$\vartheta(z, n, k)=\sum_{i=k+1}^{n}[\operatorname{SST}(i)-\operatorname{SST}(i-1)] \psi(z, n-i+1)$.

We select the GISS average global ocean surface temperatures (GISS, 2010) shown in Fig. 3 as the input data in the

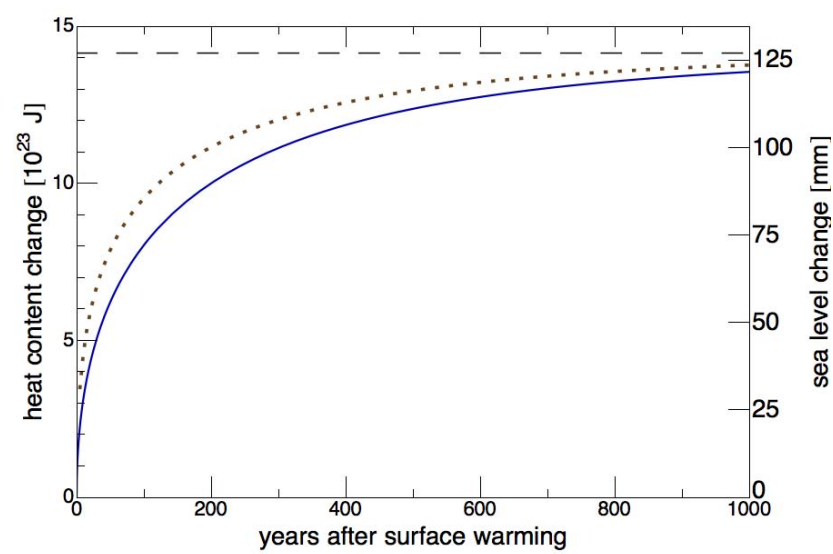

Fig. 2. Full line: heat content change of the global ocean following a $1{ }^{\circ} \mathrm{C}$ step increase of the surface temperature (left-hand scale); dotted line: the corresponding sea level change (right-hand scale).

evaluation. Although temperatures in the GISS data set fluctuate from year to year, we do not apply smoothing as the integration process itself provides sufficient averaging. The alternative global sea surface temperature dataset HadCRUT3 (CRU, 2009) is similar but shows somewhat greater variability due to a different interpolation method. Repeating the calculations with this dataset leads to similar results (not shown).

The warming of the global ocean calculated from the sea surface temperature data using Eq. (5) is illustrated in Fig. 4. In this and all the following figures, the effect of surface temperature changes before 1880 is not included in the calculation, which is equivalent to the assumption that the climate was then in steady state.

Integrating over finite depth ranges as in Eq. (3) of the previous section leads to the corresponding changes in ocean heat content due to the surface temperature changes since 1880. The integration can still be performed analytically. The result for the upper $700 \mathrm{~m}$ is shown in Fig. 5 together with two estimates based on the comprehensive review and evaluation of all available in situ measurements by Domingues et al. (2008) and Levitus et al. (2009). The calculation has no adjustable parameters other than the choice of the reference zero. For Domingues et al. (2008) and also for the calculated values, this was selected by matching the average value to Levitus et al. (2009) in the period where evaluations overlap. The heat content change calculated for the depths $700-5000 \mathrm{~m}$ is relatively small, as the surface change takes a long time to reach greater depths (see Fig. 1).

Using the average depth-dependent thermal expansion coefficient as in Fig. 2 leads to the estimated sea level change shown in Fig. 6 . The position of zero is chosen by matching the average value in the period 1950-2003. 


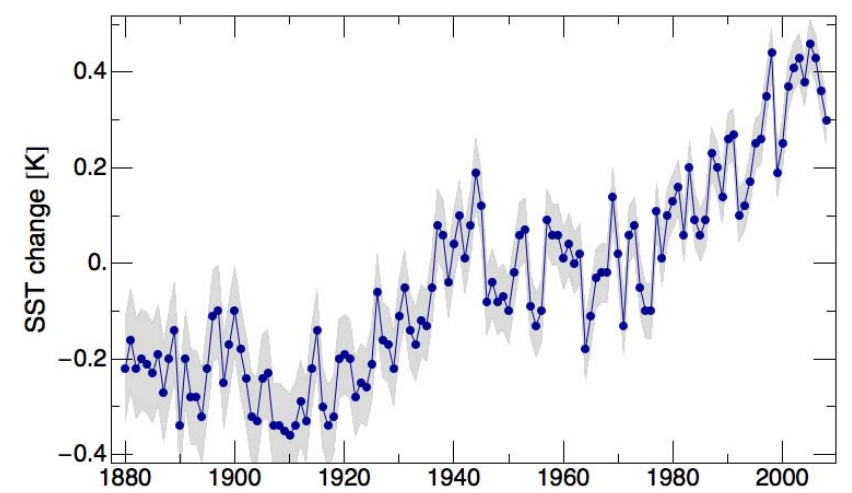

Fig. 3. NASA GISS ocean surface temperatures (GISS, 2010) used as the input in this study. The estimated $2 \sigma$ error in the data (shown as shading) decreases from $0.1 \mathrm{~K}$ at the beginning of 20th century to $0.05 \mathrm{~K}$ in recent times (Hansen et al., 2006). In this and the subsequent figures, errors in the model results that originate from the errors in the input sea surface temperatures are not shown, because random errors mostly cancel in integration and systematic errors are not known.

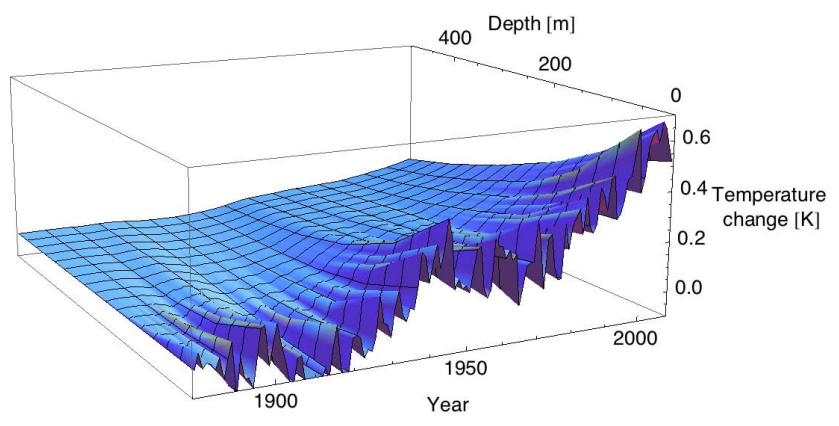

Fig. 4. Average temperature change in the top $500 \mathrm{~m}$ of the oceans resulting from surface temperature changes since 1880. At the deeper end of the figure annual variations are averaged out but decadal variations are still visible.

\section{Discussion}

Use of the advection-diffusion model of Eq. (1) to calculate the average ocean heat content and associated changes in sea level is supported by the results shown in the previous section. The major evidence comes in the agreement with the heat content $0-700 \mathrm{~m}$ calculated from the average sea surface temperature by the direct estimates available for the recent past (Domingues et al., 2008; Levitus et al., 2009). The sea level change is also in very good agreement with the earlier estimate for 0-700 $\mathrm{m}$ (Fig. 6). It is remarkable that the agreement is obtained without adjustable parameters, using the average values for eddy diffusivity and upward drift velocity determined by Munk (1966). It should also be noted that the diffusivity and drift velocity values were determined from data taken at larger depths, while this analysis indicates that they are also applicable in the $0-700 \mathrm{~m}$ range, where most of

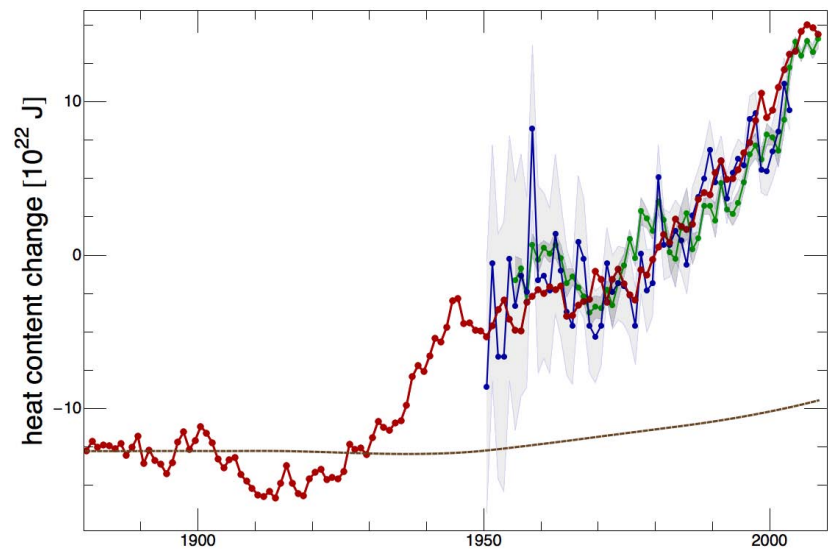

Fig. 5. Ocean heat content change over the depths $0-700 \mathrm{~m}$ calculated here from sea surface temperatures (red) and estimated using in situ measurements: Domingues et al., 2008 (blue), Levitus et al., 2009 (green). All curves show annual values positioned in the middle of the year. The dashed line at the bottom shows the calculated heat content change for depths greater than $700 \mathrm{~m}$.

the warming is observed. Average sea level rise in the period 1961-2003 atributed to thermal expansion was calculated as $0.49 \mathrm{~mm} /$ year, which can be compared to the IPCC estimate of $0.42 \pm 0.12 \mathrm{~mm} /$ year (Bindoff et al., 2007, Table 5.3).

The sensitivity of the agreement with the direct estimates was tested by varying the diffusivity and drift velocity parameters away from Munk values. To measure the goodness of fit, we used the estimate of either Domingues et al. (2008) or Levitus et al. (2009) when only one is available, and the average of the two over the 1955-2003 period when both are available. Minimising the rms difference between the calculated and the directly estimated values we found $A_{z} \approx 0.9 \times 10^{-4} \mathrm{~m}^{2} / \mathrm{s}$ instead of $A_{z} \approx 1.3 \times 10^{-4} \mathrm{~m}^{2} / \mathrm{s}$ while the drift velocity value of $W \approx 1.2 \mathrm{~cm} /$ day remained unchanged. The decrease of rms error was $12 \%$. However, the rms difference between Domingues et al. (2008) and Levitus et al. (2009) is not smaller than the rms difference between the calculated and directly estimated values. Given the present level of accuracy in the heat content measurement and modeling an improvement of the fit of this order does not justify introducing adjustable parameters by varying the empirical Munk values.

The generally good agreement of the model with the directly estimated heat content over the 58-year period 1950 2008 suggests that during that time there were no major changes in the values of average parameters $A_{z}$ and $W$. The lack of detailed agreement on the annual timescale may reflect both the limitation of the simple model that ignores variations of the global mean vertical velocity and diffusion and the limiting accuracy of the currently best estimates of changes in the global ocean heat content. 


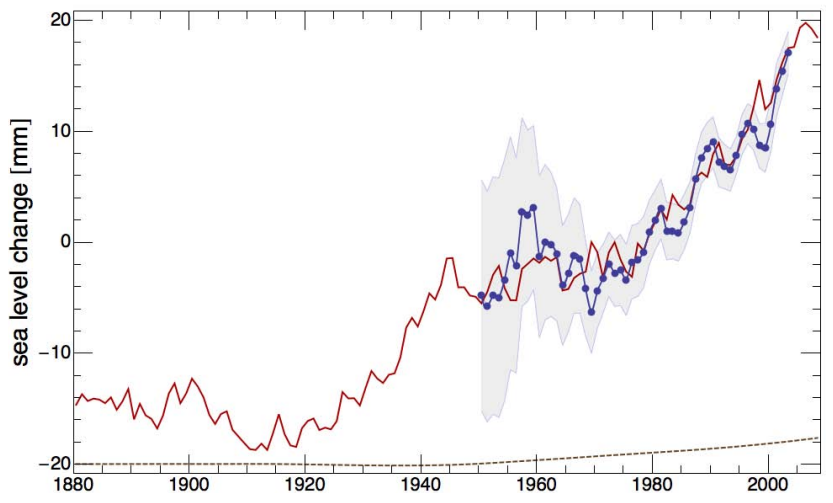

Fig. 6. Thermosteric sea level changes resulting from heat changes in the depths $0-700 \mathrm{~m}$ as calculated here from annual sea surface temperatures are shown in red. Changes calculated from in situ measurements (Domingues et al., 2008) are shown in blue as a 3 -year average with one $\sigma$ error. The dashed line at the bottom shows the calculated thermosteric contribution from depths larger than $700 \mathrm{~m}$.

The results presented include only the effects driven by the surface warming since 1880 . Estimates based on measurements (Domingues et al., 2008) show that by far the largest contribution to thermosteric sea level change originates from the upper $300 \mathrm{~m}$, where the equilibration time scale is of the order of 50 years. Global surface temperature reconstructions do not indicate major changes over the $1830-1880$ period (Jansen et al., 2007; Jones and Mann, 2004) and the evaluation beginning in 1880 is probably adequate to describe most of the effect in the upper ocean. The initial period in the reconstructions presented here is nevertheless subject to more uncertainty.

The warming of the deep ocean is much slower, and earlier changes in the global climate may still be spreading to deeper layers. The sea level change originating from the ocean warming below $700 \mathrm{~m}$ evaluated here is small, because of both the limited warming and small expansion coefficient. We estimate about $0.04 \mathrm{~mm} / \mathrm{yr}$ for the most recent 50 years. This value does not agree with $0.20 \pm 0.1 \mathrm{~mm} / \mathrm{yr}$ conjectured by Domingues et al. (2008).

The steady upward drift in the Munk model is balanced by the downwelling flows, which may have varied in heat content during the analysis period. The abyssal heat budget is dominated by flows at the boundaries rather than deep diffusivity (Scott and Marotzke, 2002, and references therein). The contributions resulting from the changes in downwelling flows, which are neglected in the present model, are important to the overall heat budget, even though they have a less pronounced effect on the sea level change. Promising new methods combining space gravimetry with altimetric measurements (see e.g. Cazenave et al., 2009) will provide more insight into the closure of the sea level change budget.

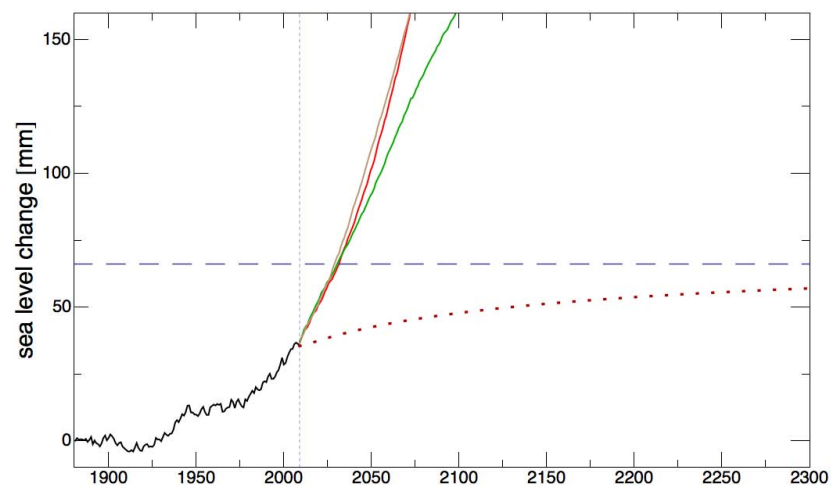

Fig. 7. Dotted line: future projection of the thermal expansion contribution to sea level rise based on the formal assumption that sea surface temperature will not increase beyond the changes of 18802008. Projections for the thermal expansion contribution to sea level rise corresponding to IPCC future climate scenarios (starting in 2009) are shown as red (A2), brown (A1B) and green (B1) lines. They are not far from the average projections for the rise obtained from more complex climate models (Meehl et al., 2007, Fig. 10.31).

The method of calculating average ocean heat content and the associated sea level change using only surface temperatures opens up the possibility of expanding our knowledge into the past and future. In order to illustrate the timescale of the sea level response we show in Fig. 7 the unrealistic scenario where all warming of the sea surface had stopped and the present average temperature is maintained into the future. The figure shows the slow rate of the approach to the steady state value. In this example we had $35 \mathrm{~mm}$ rise since 1880 and we are committed to a further $30 \mathrm{~mm}$. These increases are listed only as an illustration, and the present level of greenhouse gasses in the atmosphere already leads to a commitment for significant future warming (Meehl et al., 2007, Fig. 10.4).

A more realistic projection can be obtained using IPCC scenarios A2 ("high"), A1B ("medium") and B1 ("low"). Global temperatures until 2100 were taken as the climate model averages provided by IPCC (Meehl et al., 2007, Fig. 10.5) and aligned with the measured sea surface temperature by matching the averages in the period 2000-2008. The contribution of thermal expansion to changes in sea level was calculated using these composite 1880-2100 temperatures as the input (Fig. 7). In order to compare the results with evaluations from climate models we use the IPCC methodology and calculate the average thermal expansion between 19801999 and 2090-2099 periods. The results are (with the IPCC range in parentheses, Meehl et al., 2007, Table 10.7) A2: $0.21 \mathrm{~m}(0.14-0.35)$, A1B: $0.19 \mathrm{~m}(0.13-0.32), \mathrm{B} 1: 0.13 \mathrm{~m}$ $(0.10-0.24)$.

In summary, we show that a simple global average calculation of ocean warming based on the advection-diffusion model and using the values of the coefficients determined 
in the past from steady-state measurements accurately reproduces much more detailed recent data. In the amount of input information required, there is a vast difference between the average and detailed calculations. The method described, which uses only global averages, provides a useful tool to understand the past heat content and associated sea level changes and to project the effect of present-day surface temperature changes into the future.

Acknowledgements. Joe Wolfe offered valuable advice and criticism and Neil White kindly made available detailed data on heat content and sea level change used in Figs. 5-6. Valuable suggestions from the reviewers are gratefully acknowledged.

Edited by: A. Sterl

\section{References}

Bindoff, N. L., Willebrand, J., Artale, V., Cazenave, A., Gregory, J., Gulev, S., Hanawa, K., Le Quéré, C., Levitus, S., Nojiri, Y., Shum, C. K., Talley L. D., and Unnikrishnan, A.: Observations: Oceanic climate change and sea level, in: Climate change 2007: The physical science basis, Cambridge University Press, Cambridge, 2007.

Cazenave, A., Dominh, K., Guinehut, S., Berthier, E., Llovel, W., Ramillien, G., Ablain, M., and Larnicol, G.: Sea level budget over 2003-2008: A reevaluation from GRACE space gravimetry, satellite altimetry and Argo, Global Planet. Change, 65, 83-88, 2009.

Domingues, C. M., Church, J. A., White, N. J., Gleckler, P. J., Wijffels, S. E., Barker, P. M., and Dunn, J. R.: Improved estimates of upper-ocean warming and multi-decadal sea-level rise, Nature, 453, 1090-1093, 2008.

GISS 2009: online available at: http://data.giss.nasa.gov/gistemp/ time_series.html, last access: February 2010.

Grinsted, A., Moore, J. C., and Jevrejeva, S.: Reconstructing sea level from paleo and projected temperatures 200 to $2100 \mathrm{AD}$, Clim. Dynam., doi:10.1007/s00382-008-0507-2, 2009.

Hansen, J., Sato, M., Ruedy, R., Lo, K., Lea, D. W., and MedinaElizade, M.: Global temperature change, P. Natl. Acad. Sci., 103, 14288-14293, 2006.
Jansen, E., Overpeck, J., Briffa, K. R., Duplessy, J.-C., Joos, F., Masson-Delmotte, V., Olago, D., Otto-Bliesner, B., Peltier, W. R., Rahmstorf, S., Ramesh, R., Raynaud, D., Rind, D., Solomina, O., Villalba, R., and Zhang, D.: Palaeoclimate, in Climate change 2007: The physical science basis, Cambridge University Press, Cambridge, 2007.

Jones, P. D. and Mann, M. E.: Climate over past millenia, Rev. Geophys., 42, RG2002, doi:10.1029/2003RG000143, 2004.

Lee, T.-C.: Applied mathematics in hydrogeology, A. F. Lewis, New York, 1999.

Levitus, S.: Climatological atlas of the world ocean, NOAA Prof. Paper 13, US Govt., Printing Office, Washington DC, 1982.

Levitus, S., Antonov, J. I., Boyer, T. P., Locarnini, R. A., Garcia, H. E., and Mishonov, A. V.: Global ocean heat content 1955-2008 in light of recently revealed instrumentation problems, Geophys. Res. Lett., 36, L07608, doi:10.1029/2008GL037155, 2009.

McDougall, T. J., Jackett, D. R., Wright, D. G., and Feistel, R.: Accurate and computationally efficient algorithms for potential temperature and density of seawater, J. Atmos. Ocean. Tech., 20, 730-741, 2003.

Meehl, G. A., Stocker, T. F., Collins, W. D., Friedlingstein, P., Gaye, A. T., Gregory, J. M., Kitoh, A., Knutti, R., Murphy, J. M., Noda, A., Raper, S. C. B., Watterson, I. G., Weaver, A. J., and Zhao, Z.C.: Global climate projections, in: Climate change 2007: The physical science basis, Cambridge University Press, Cambridge, 2007.

Munk, W. H.: Abyssal recipes, Deep-Sea Res., 13, 707-730, 1966.

Ogata, A.: Theory of dispersion in a granular medium, Technical report, US Geol. Surv. Prof. Paper, 411-I, I1-I34, 1970.

Rahmstorf, S.: A semi-empirical approach to projecting future sealevel rise, Science, 315, 368-370, 2007.

Scott, J. R. and Marotzke, J.: The location of diapycnal mixing and the meridional overturning circulation, J. Phys. Oceanogr., 32, 3578-3595, 2002.

Siddall, M., Stocker, T. F., and Clark, P. U.: Constraints on future sea-level rise from past sea-level change, Nature Geoscience, 2, 571-575, 2009.

Stewart, R. H.: Introduction to physical oceanography, online available at: http://oceanworld.tamu.edu/home/course_book.htm, last access: November 2009. 